\title{
Scale-Dependent Non-Gaussianity as a Generalization of the Local Model
}

\author{
Adam Becker, Dragan Huterer, Kenji Kadota \\ Department of Physics and Michigan Center for Theoretical Physics \\ University of Michigan, 450 Church Street, Ann Arbor, MI 48109
}

\begin{abstract}
We generalize the local model of primordial non-Gaussianity by promoting the parameter $f_{\mathrm{NL}}$ to a general scale-dependent function $f_{\mathrm{NL}}(k)$. We calculate the resulting bispectrum and the effect on the bias of dark matter halos, and thus the extent to which $f_{\mathrm{NL}}(k)$ can be measured from the large-scale structure observations. By calculating the principal components of $f_{\mathrm{NL}}(k)$, we identify scales where this form of non-Gaussianity is best constrained and estimate the overlap with previously studied local and equilateral non-Gaussian models.
\end{abstract}

Keywords: Cosmology. 


\section{Contents}

1. Introduction 1

2. Scale dependent non-Gaussianity 2

3. Non-Gaussianity and Bias 3

3.1 The effect of a non-vanishing bispectrum on bias 3

3.2 From the bispectrum to bias 5

3.2.1 Constant $f_{\mathrm{NL}} \quad 5$

$\begin{array}{lll}3.2 .2 & \text { Scale-dependent } f_{\mathrm{NL}} & 6\end{array}$

4. Forecasted measurements of the scale-dependent nongaussianity 7

$\begin{array}{lll}4.1 & \text { Fisher Matrix Analysis } & 7\end{array}$

5. Projection and Principal Components 9

5.1 Constraining other $f_{\mathrm{NL}}(k)$ models 9

5.2 Principal components and relation to local and equilateral models $\quad 11$

$\begin{array}{lll}\text { 6. Conclusions } & 14\end{array}$

$\begin{array}{ll}\text { 7. Acknowledgements } & 15\end{array}$

$\begin{array}{ll}\text { A. Calculating the error on an arbitrary parametrized } f_{\mathrm{NL}}(k) & 15\end{array}$

B. Principal Components of $f_{\mathrm{NL}}(k) \quad 16$

C. Generalized local ansatz does not recover the equilateral case $\quad 17$

\section{Introduction}

Primordial non-Gaussianity provides cosmology one of the precious few connections between primordial physics and the present-day universe. Standard inflationary theory, with a single slowly rolling scalar field, predicts that the spatial distribution of structures in the universe today is very nearly Gaussian random (e.g. [1, 2, 3, 4, 5]; for excellent recent reviews, see $[6,7])$. Departures from Gaussianity, barring contamination from systematic errors or late-time non-Gaussianity due to secondary processes, would be a violation of this standard inflationary assumption. Constraining or detecting primordial non-Gaussianity is therefore an important basic test of the standard cosmological model.

Most of the study of non-Gaussianity in the literature to date has been carried out assuming the magnitude of departure from Gaussianity is scale-independent (e.g. [8, 9, 10]). 
However, the assumption that $f_{\mathrm{NL}}$ is constant for a wide range of scales could be an oversimplification, since the primordial cosmic perturbations were presumably produced from the time-dependent dynamics in the early universe. In particular, single-field inflationary models with interactions, along with most multi-field models, generically produce scaledependent non-Gaussianity. It is therefore not surprising that scale-dependence of nonGaussianity has been discussed in the community in recent years $[11,12,13,14,15,16$, $17,18,19,20,21,22,23,24,25,26,27,28,29]$. Notably, the parameterization of the scale-dependent non-Gaussianity in our analysis is applicable to the curvaton [30, 31, 32, $33,34]$ and the modulated reheating scenarios [35, 36], which are of great interest for their potentially observable scale-dependent non-Gaussianity ${ }^{1}$.

Motivated by such inflationary models that predict detectable scale-dependent nonGaussianity, as well as a desire to have an easily usable basis for studying those models, we present a novel scale-dependent ansatz for primordial non-Gaussianity: we promote the parameter $f_{\mathrm{NL}}$ to a free function of wavenumber $f_{\mathrm{NL}}(k)$. We define our model (Sec. 2), predict clustering bias of dark matter halos in our model (Sec. 3), obtain an upper bound on the accuracy with which these new parameters could be measured with a future largescale structure survey (Sec. 4), and compare our model with other parameterizations of non-Gaussianity in the literature (Sec. 5).

\section{Scale dependent non-Gaussianity}

The most commonly discussed model of non-Gaussianity, often referred to as the local model, is defined via [8]

$$
\Phi(x)=\phi_{G}(x)+f_{\mathrm{NL}}\left(\phi_{G}(x)^{2}-\left\langle\phi_{G}(x)^{2}\right\rangle\right) .
$$

Here, $\Phi$ denotes the primordial curvature perturbations (Bardeen's gauge-invariant potential), $\phi_{G}(x)$ is a Gaussian random field, and the constant $f_{\mathrm{NL}}$ is the non-Gaussianity parameter. The local model has been much studied, in part because it is the first two terms of the most general local form of non-Gaussianity [40].

In Fourier space, Eq. (2.1) becomes

$$
\Phi(k)=\phi_{G}(k)+f_{\mathrm{NL}} \int \frac{d^{3} k^{\prime}}{(2 \pi)^{3}} \phi_{G}\left(k^{\prime}\right) \phi_{G}\left(k-k^{\prime}\right) .
$$

(Hereafter, we omit the subscript $G$ on the Gaussian distribution when it is clear from context.) In this paper, we study a model that generalizes Eq. $(2.2)$ - we allow $f_{\mathrm{NL}}$ to vary with $k$ as well, while assuming isotropy and homogeneity (so $f_{\mathrm{NL}}(\boldsymbol{k})=f_{\mathrm{NL}}(k)$ ). The

\footnotetext{
${ }^{1}$ For instance, when the observed perturbations originate from the single curvaton field, the "running" (with scale) of the non-Gaussianity parameter is proportional to the third derivative of the curvaton potential, $V^{\prime \prime \prime}[37,38,39]$. Given that this third derivative is not tightly constrained from the observed power spectrum, it can potentially lead to observable and scale-dependent non-Gaussianity. Therefore, constraints on the running of non-Gaussianity can be a powerful probe of the origin of the primordial curvature perturbations.
} 
gravitational potential in the new model is defined via

$$
\Phi(k)=\phi(k)+f_{\mathrm{NL}}(k) \int \frac{d^{3} k^{\prime}}{(2 \pi)^{3}} \phi\left(k^{\prime}\right) \phi\left(k-k^{\prime}\right) .
$$

As mentioned above, this form of non-Gaussianity is expected in curvaton or modulated reheating scenarios (see e.g. Ref. [37], where this form explicitly appears in the study of these models).

Note that this new ansatz is not local, which is clear when we transform back into real space:

$$
\Phi(x)=\phi+f_{\mathrm{NL}}(x) *\left(\phi(x)^{2}-\left\langle\phi(x)^{2}\right\rangle\right),
$$

where $*$ represents convolution and $x$ denotes a three-dimensional spatial coordinate. These primordial perturbations $\Phi(k)$ are related to the present-time $(\mathrm{z}=0)$ smoothed linear overdensity $\delta_{R}$ by the Poisson equation:

$$
\delta_{R}(k)=\frac{2}{3} \frac{k^{2} T(k)}{H_{0}^{2} \Omega_{m}} \tilde{W}_{R}(k) \Phi(k) \equiv \mathcal{M}_{R}(k) \Phi(k) ;
$$

where $T(k)$ is the matter transfer function, $H_{0}$ is the Hubble constant, $\Omega_{m}$ is the matter density relative to critical today, and $\tilde{W}_{R}(k)$ is the Fourier transform of the top-hat filter with radius $R$. The smoothing spatial scale $R$ is related to the smoothing mass scale $M$ via

$$
M=\frac{4}{3} \pi R^{3} \rho_{m, 0},
$$

where $\rho_{m, 0}$ is the matter energy density today. The choice of mass scale is discussed further in section 4.1.

The bispectrum in our generalized model becomes

$$
B_{\phi}\left(k_{1}, k_{2}, k_{3}\right)=2\left[f_{\mathrm{NL}}\left(k_{1}\right) P_{\phi}\left(k_{2}\right) P_{\phi}\left(k_{3}\right)+\text { perm. }\right],
$$

where $P_{\phi}$ is the power spectrum of potential fluctuations. This reduces to the familiar expression $B\left(k_{1}, k_{2}, k_{3}\right)=2 f_{\mathrm{NL}}\left(P_{\phi}\left(k_{1}\right) P_{\phi}\left(k_{2}\right)+\right.$ perm. $)$ when $f_{\mathrm{NL}}$ is a constant.

Notice the difference between our ansatz for the scale-dependent $f_{\mathrm{NL}}(k)$ (which has the corresponding bispectrum Eq. (2.7)) and the particular form of scale-dependent nonGaussianity, discussed elsewhere in the literature, which is defined as $f_{\mathrm{NL}}\left(k_{1}, k_{2}, k_{3}\right) \equiv$ $B_{\phi}\left(k_{1}, k_{2}, k_{3}\right) /\left[2 P_{\phi}\left(k_{1}\right) P_{\phi}\left(k_{2}\right)+\right.$ perm. $]([25,27,26])$. The two forms are inequivalent, and either form can be borne out in realistic inflationary models; however, given that our form lives in a lower-dimensional $k$-space, it is easier to simulate it numerically [41] or treat it with the Fisher matrix analysis, as we do in this paper.

\section{Non-Gaussianity and Bias}

\subsection{The effect of a non-vanishing bispectrum on bias}

Dalal et al. [42] found, analytically and numerically, that the bias of dark matter halos acquires strong scale dependence if $f_{\mathrm{NL}} \neq 0$ :

$$
b(k)=b_{0}+f_{\mathrm{NL}}\left(b_{0}-1\right) \delta_{c} \frac{3 \Omega_{m} H_{0}^{2}}{a g(a) T(k) c^{2} k^{2}} .
$$


Here, $b_{0}$ is the usual Gaussian bias (on large scales, where it is constant), $\delta_{c} \approx 1.686$ is the collapse threshold, $a$ is the scale factor, $\Omega_{m}$ is the matter density relative to the critical density, $H_{0}$ is the Hubble constant, $k$ is the wavenumber, $T(k)$ is the transfer function, and $g(a)$ is the growth suppression factor ${ }^{2}$. This result has been confirmed by other researchers using a variety of methods, including the peak-background split [43, 44, 45, 46], perturbation theory [47, 48, 49], and numerical (N-body) simulations [50, 51, 52]. Astrophysical measurements of the scale dependence of the large-scale bias, using galaxy and quasar clustering as well as the cross-correlation between the galaxy density and CMB anisotropy, have recently been used to impose constraints on $f_{\mathrm{NL}}$ already comparable to those from the cosmic microwave background (CMB) anisotropy [45, 43], giving $f_{\mathrm{NL}}=$ $28 \pm 23(1 \sigma)$, with some dependence on the assumptions made in the analysis [45]. In the future, constraints on $f_{\mathrm{NL}}$ are expected to be on the order of a few $[42,53,54,55]$. The sensitivity of the large-scale bias to other models of primordial non-Gaussianity has not yet been investigated much (though see analyses in e.g. [56, 57]).

Following the MLB formula $[58,59]$, one can express the two point correlation function of dark matter halos, $\xi_{h}\left(\boldsymbol{x}_{1}, \boldsymbol{x}_{2}\right)$, in terms of certain configurations of the correlation functions of the underlying density field, $\xi_{R}^{(N)}$. In the high-threshold limit $(\nu \gg 1)$, this becomes:

$$
\begin{aligned}
\xi_{h}\left(\boldsymbol{x}_{1}, \boldsymbol{x}_{2}\right) & =\xi_{h}\left(x_{12}\right) \\
& =-1+\exp \left(\sum_{N=2}^{\infty} \sum_{j=1}^{N-1} \frac{\nu^{N}}{\sigma_{R}^{N}} \frac{1}{j !(N-j) !} \xi_{R}^{(N)}\left[\begin{array}{cc}
\boldsymbol{x}_{1}, \ldots, \boldsymbol{x}_{1}, & \boldsymbol{x}_{2}, \ldots, \boldsymbol{x}_{2} \\
j \text { times } & (N-j) \text { times }
\end{array}\right]\right)(;
\end{aligned}
$$

where $x_{i j}=\left|\boldsymbol{x}_{i}-\boldsymbol{x}_{j}\right|, \nu=\delta_{c} / \sigma_{R}$ represents the peak height, and $\xi_{R}^{(n)}(r)$ is the $n$-point correlation function of the underlying matter density smoothed with a top-hat filter of radius $R$. Keeping the terms up to the three-point correlation function, which would be reasonable for the observationally allowed range of $f_{\mathrm{NL}}$, the expansion series gives us the halo correlation function in terms of the field correlation functions:

$$
\xi_{h}\left(x_{12}\right)=\frac{\nu^{2}}{\sigma_{R}^{2}} \xi_{R}^{(2)}\left(\boldsymbol{x}_{1}, \boldsymbol{x}_{2}\right)+\frac{\nu^{3}}{\sigma_{R}^{3}} \xi_{R}^{(3)}\left(\boldsymbol{x}_{1}, \boldsymbol{x}_{1}, \boldsymbol{x}_{2}\right) .
$$

The Fourier transform of the real-space correlation function - the power spectrum is given, to the same expansion order as Eq. (3.3), by

$$
P_{h}(k)=\frac{\nu^{2}}{\sigma_{R}^{2}} P_{R}(k)+\frac{\nu^{3}}{\sigma_{R}^{3}} \int \frac{d^{3} q}{(2 \pi)^{3}} B_{R}(k, q,|\boldsymbol{k}-\boldsymbol{q}|)+\ldots
$$

The first term on the right-hand side includes the familiar (Gaussian) bias $b=\nu / \sigma_{R}$ (in the high-peak limit for which the MLB formula is valid) for the Gaussian fluctuations. The effects of non-Gaussianity on the galaxy bias are represented by the second term, including the bispectrum $B_{R}$, which vanishes for the Gaussian fluctuations.

\footnotetext{
${ }^{2}$ The usual linear growth $D(a)$, normalized to be equal to $a$ in the matter-dominated epoch, is related to the suppression factor $g(a)$ via $D(a)=a g(a)$, where $g(a)$ is normalized to be equal to unity deep in the matter-dominated epoch.
} 


\subsection{From the bispectrum to bias}

If we denote the full bias of dark matter halos by $b+\Delta b$, where $b$ represents the bias for the Gaussian fluctuations and $\Delta b$ is the non-Gaussian correction, then

$$
\frac{P_{h}}{P_{R}}=b^{2}\left(1+\frac{\Delta b}{b}\right)^{2},
$$

where $P_{h}$ and $P_{R}$ are the power spectra of halos and dark matter, respectively. The nonGaussian correction to the linear peak bias to the leading order becomes

$$
\frac{\Delta b}{b}(k)=\frac{\nu}{\sigma_{R}} \frac{1}{2 P_{R}(k)} \int \frac{d^{3} q}{(2 \pi)^{3}} B_{R}(k, q,|\boldsymbol{k}-\boldsymbol{q}|),
$$

where $B_{R}$ is the matter bispectrum on scale $R$. Hence, the non-Gaussian correction $\Delta b(k)$ can be expressed in terms of the primordial potential fluctuations as ([44]):

$$
\frac{\Delta b}{b}(k)=\frac{\delta_{c}}{D(z)} \frac{1}{8 \pi^{2} \sigma_{R}^{2} \mathcal{M}_{R}(k)} \int_{0}^{\infty} d k_{1} k_{1}^{2} \mathcal{M}_{R}\left(k_{1}\right) \int_{-1}^{1} d \mu \mathcal{M}_{R}\left(k_{2}\right) \frac{B_{\phi}\left(k_{1}, k_{2}, k\right)}{P_{\phi}(k)} .
$$

We perform the integration over all triangles. The triangles' sides are $k_{1}, k_{2}$, and $k$; the cosine of the angle opposite $k_{2}$ is $\mu$, so $k_{2}^{2}=k_{1}^{2}+k^{2}+2 k_{1} k \mu . \mathcal{M}_{R}(k)$ is the same function defined in Eq. (2.5), and the time dependence of the critical threshold for collapse is given as $\delta_{c}(z)=\delta_{c} / D(z)$, with $\delta_{c}=1.686$.

\subsubsection{Constant $f_{\mathrm{NL}}$}

Eq. (3.7) leads to the famous scale-dependent bias formula in the case of a constant $f_{\mathrm{NL}}$. For this model, the bispectrum is

$$
B_{\phi}\left(k_{1}, k_{2}, k_{3}\right)=2 f_{\mathrm{NL}}\left[P_{\phi}\left(k_{1}\right) P_{\phi}\left(k_{2}\right)+\text { perm. }\right] .
$$

Through Eq. (3.7), this leads to the result

$$
\begin{aligned}
\frac{\Delta b}{b}(k) & =\frac{\delta_{c}}{D(z)} \frac{2 f_{\mathrm{NL}}}{8 \pi^{2} \sigma_{R}^{2} \mathcal{M}_{R}(k)} \int d k_{1} k_{1}^{2} \mathcal{M}_{R}\left(k_{1}\right) P_{\phi}\left(k_{1}\right) \int d \mu \mathcal{M}_{R}\left(k_{2}\right)\left[\frac{P_{\phi}\left(k_{2}\right)}{P_{\phi}(k)}+2\right] \\
& \equiv \frac{2 f_{\mathrm{NL}} \delta_{c}}{D(z)} \frac{\mathcal{F}(k)}{\mathcal{M}_{R}(k)},
\end{aligned}
$$

where

$$
\mathcal{F}(k) \equiv \frac{1}{8 \pi^{2} \sigma_{R}^{2}} \int d k_{1} k_{1}^{2} \mathcal{M}_{R}\left(k_{1}\right) P_{\phi}\left(k_{1}\right) \int d \mu \mathcal{M}_{R}\left(k_{2}\right)\left[\frac{P_{\phi}\left(k_{2}\right)}{P_{\phi}(k)}+2\right] .
$$

Note that there is a factor of 2 in Eq. (3.9) because we can exchange the order of integration of terms corresponding to $k_{1}$ and $k_{2}$.

Finally, we rewrite Eq. (3.9) by defining

$$
\begin{aligned}
& \mathcal{F}_{1}(k) \equiv \frac{1}{8 \pi^{2} \sigma_{R}^{2} \mathcal{M}_{R}(k) P_{\phi}(k)} \int d k_{1} k_{1}^{2} \mathcal{M}_{R}\left(k_{1}\right) P_{\phi}\left(k_{1}\right) \int d \mu \mathcal{M}_{R}\left(k_{2}\right) P_{\phi}\left(k_{2}\right) \\
& \mathcal{F}_{2}(k) \equiv \frac{2}{8 \pi^{2} \sigma_{R}^{2} \mathcal{M}_{R}(k)} \int d k_{1} k_{1}^{2} \mathcal{M}_{R}\left(k_{1}\right) P_{\phi}\left(k_{1}\right) \int d \mu \mathcal{M}_{R}\left(k_{2}\right) .
\end{aligned}
$$


Then, for constant $f_{\mathrm{NL}}$,

$$
\frac{\Delta b}{b}(k)=\frac{2 f_{\mathrm{NL}} \delta_{c}}{D(z)}\left[\mathcal{F}_{1}(k)+\mathcal{F}_{2}(k)\right]
$$

and the derivative with respect to $f_{\mathrm{NL}}$ is

$$
\frac{\partial}{\partial f_{\mathrm{NL}}}\left[\frac{\Delta b}{b}(k)\right]=\frac{2 \delta_{c}}{D(z)}\left[\mathcal{F}_{1}(k)+\mathcal{F}_{2}(k)\right] .
$$

\subsubsection{Scale-dependent $f_{\mathrm{NL}}$}

Now we repeat the analysis of the previous section, but we allow $f_{\mathrm{NL}}(k)$ to be an arbitrary function of scale, adopting the ansatz in Eq. (2.3). We still assume homogeneity, so $f_{\mathrm{NL}}(\vec{k})=f_{\mathrm{NL}}(k)$. The bispectrum is given by

$$
B_{\phi}\left(k_{1}, k_{2}, k_{3}\right)=2\left[f_{\mathrm{NL}}\left(k_{1}\right) P_{\phi}\left(k_{2}\right) P_{\phi}\left(k_{3}\right)+\text { perm. }\right] .
$$

Here, the triangle condition always holds, so that (for example) $k_{1}=\left|\overrightarrow{k_{2}}+\overrightarrow{k_{3}}\right|$. Following Eq. (3.7), we get

$$
\begin{aligned}
\frac{\Delta b}{b}(k) & =\frac{\delta_{c}}{D(z)} \frac{2}{8 \pi^{2} \sigma_{R}^{2} \mathcal{M}_{R}(k)} \int d k_{1} k_{1}^{2} \mathcal{M}_{R}\left(k_{1}\right) P_{\phi}\left(k_{1}\right) \\
& \times \int d \mu \mathcal{M}_{R}\left(k_{2}\right)\left[f_{\mathrm{NL}}(k) \frac{P_{\phi}\left(k_{2}\right)}{P_{\phi}(k)}+2 f_{\mathrm{NL}}\left(k_{2}\right)\right] .
\end{aligned}
$$

This looks like Eq. (3.9) - but this time, $f_{\mathrm{NL}}(k)$ is a function, not a constant. Thus, to find the derivative of $\Delta b / b(k)$ with respect to the relevant parameters, we must parametrize $f_{\mathrm{NL}}(k)$ in a way that is valid for any general form of $f_{\mathrm{NL}}(k)$. We consider the piecewiseconstant (in wavenumber) parametrization where $f_{\mathrm{NL}}(k)$ is equal to $f_{\mathrm{NL}}^{i}$ in the $i$ th wavenumber bin:

$$
f_{\mathrm{NL}}^{i} \equiv f_{\mathrm{NL}}\left(k_{i}\right)
$$

The derivative of $\Delta b / b(k)$ with respect to these $f_{\mathrm{NL}}^{i}$ is:

$$
\begin{aligned}
\frac{\partial}{\partial f_{\mathrm{NL}}^{j}}\left[\frac{\Delta b}{b}\left(k_{i}\right)\right]= & \frac{\delta_{c}}{D(z)} \frac{2}{8 \pi^{2} \sigma_{R}^{2} \mathcal{M}_{R}(k)} \times \\
& {\left[\delta_{i j} \frac{1}{P_{\phi}(k)} \int d k_{1} k_{1}^{2} \mathcal{M}_{R}\left(k_{1}\right) P_{\phi}\left(k_{1}\right) \int d \mu \mathcal{M}_{R}\left(k_{2}\right) P_{\phi}\left(k_{2}\right)+\right.} \\
& \left.+2 \int_{k_{2} \in k_{j}} d k_{1} k_{1}^{2} \mathcal{M}_{R}\left(k_{1}\right) P_{\phi}\left(k_{1}\right) \int d \mu \mathcal{M}_{R}\left(k_{2}\right)\right]
\end{aligned}
$$

where $\delta_{i j}$ is the Kronecker delta function. Note that the last integral over $k_{2}$ only goes over the $j$ th wavenumber bin.

This derivative can be rewritten more concisely as

$$
\frac{\partial}{\partial f_{\mathrm{NL}}^{j}}\left[\frac{\Delta b}{b}\left(k_{i}\right)\right]=\frac{2 \delta_{c}}{D(z)}\left[\delta_{i j} \mathcal{F}_{1}(k)+\mathcal{F}_{2}^{j}(k)\right] .
$$

The functions $\mathcal{F}_{1}$ and $\mathcal{F}_{2}$ are defined as in Eqs. (3.11) and (3.12), except that the superscript in $F_{2}^{j}$ indicates that the integral over $k_{2}$ is to be executed only over the $j$ th wavenumber bin. 


\section{Forecasted measurements of the scale-dependent nongaussianity}

\subsection{Fisher Matrix Analysis}

With an expression for $\partial / \partial f_{\mathrm{NL}}^{j}\left[(\Delta b / b)\left(k_{i}\right)\right]$ in hand (Eq. (3.19)), we can calculate the Fisher information matrix for the parameters $f_{\mathrm{NL}}^{j}$ that describe the piecewise-constant $f_{\mathrm{NL}}(k)$. The Fisher matrix, in turn, allows us to forecast the extent to which the scale-dependent non-Gaussianity could be measured in future galaxy surveys.

We consider measurements of the power spectrum $P_{h}(k)$ of dark matter halos (galaxies or clusters, for example) averaged over thin spherical shells in $k$-space. The variance of $P_{h}(k) \equiv P_{h}$ in each shell is [60]

$$
\sigma_{P_{h}}^{2}=\frac{2 P_{h}^{2}}{V_{\text {shell }} V_{\text {survey }}}\left(\frac{1+n P_{h}}{n P_{h}}\right)^{2}=\frac{\left(2 \pi P_{h}\right)^{2}}{k^{2} d k V_{\text {survey }}}\left(\frac{1+n P_{h}}{n P_{h}}\right)^{2},
$$

where $V_{\text {shell }}=4 \pi k^{2} d k /(2 \pi)^{3}$ is the volume of the shell in Fourier space (we are ignoring redshift distortion effects for simplicity here). Therefore, the Fisher matrix for measurements of $P_{h}(k, z)$ is [61]

$$
F_{i j}=\sum_{m} V_{m} \int_{k_{\min }}^{k_{\max }} \frac{\partial P_{h}\left(k, z_{m}\right)}{\partial p_{i}} \frac{\partial P_{h}\left(k, z_{m}\right)}{\partial p_{j}} \frac{1}{\left[P_{h}\left(k, z_{m}\right)+\frac{1}{n}\right]^{2}} \frac{k^{2} d k}{(2 \pi)^{2}},
$$

where $V_{m}$ is the comoving volume of the $m$-th redshift bin, each redshift bin is centered on $z_{m}$, and we have summed over all redshift bins. We adopt $k_{\min }=10^{-4} h^{-1} \mathrm{Mpc}$, and we choose $k_{\max }$ as a function of $z$ so that $\sigma\left(\pi /\left(2 k_{\max }\right), z\right)=0.5$ [62], which leads to $k_{\max }(z=0) \approx 0.1 \mathrm{~h} \mathrm{Mpc}^{-1}$. Finally, $p_{i}$ are the parameters of interest; in our case, these are the $f_{\mathrm{NL}}^{i}$.

We assume a flat universe and a fiducial model of zero non-Gaussianity: $f_{\mathrm{NL}}(k)=$ $0=f_{\mathrm{NL}}^{i}$. We include six cosmological parameters in our Fisher matrix aside from the $f_{\mathrm{NL}}^{i}$ : Hubble's constant $H_{0}$; physical dark matter and baryon densities $\Omega_{\mathrm{cdm}} h^{2}$ and $\Omega_{\mathrm{b}} h^{2}$; equation of state of dark energy $w$; the log of the scalar amplitude of the matter power spectrum, $\log A_{s}$; and the spectral index of the matter power spectrum, $n_{s}$. Fiducial values of these parameters correspond to their best-fit WMAP7 values [63]. We also added the forecasted cosmological parameter constraints from the CMB experiment Planck by adding its Fisher matrix as a prior (W. Hu, private communication). Note that the CMB prior does not include CMB constraints on non-Gaussianity; the CMB constraints on $f_{\mathrm{NL}}(k)$ will be separately studied in a future work. Finally, in addition to the cosmological parameters and the $f_{\mathrm{NL}}^{i}$, we include five Gaussian bias parameters in our Fisher matrix - one $b_{0}(z)$ for each redshift bin. The fiducial values of these parameters are set by the relations $b_{0}(z=0)=2.2$, and $b_{0}(z)=b_{0}(z=0) / D(z)$.

We already have the derivatives of $b(k)$ with respect to each of the $f_{\mathrm{NL}}^{i}$, so the derivative of $P_{h}(k)$ with respect to the $f_{\mathrm{NL}}^{i}$ is just

$$
\frac{\partial P_{h}(k)}{\partial f_{\mathrm{NL}}^{i}}=2 \frac{\partial b(k)}{\partial f_{\mathrm{NL}}^{i}} b(k) P_{\mathrm{mat}}(k) ;
$$


$P_{\text {mat }}(k)$ is the $\Lambda \mathrm{CDM}$ matter power spectrum, easily obtained from a numerical code such as CAMB. Since we only consider information from large scales $\left(k \leq k_{\max } \approx 0.1 \mathrm{~h} \mathrm{Mpc}^{-1}\right)$, we do not model the small amount of nonlinearity present at the high- $k$ end of these scales.

We assume a future survey covering one-quarter of the sky (about 10,000 square degrees) out to $z=1$, and find constraints for a set of $20 f_{\mathrm{NL}}^{i}$ uniformly spaced in $\log k$ in the range $10^{-4} \leq k /\left(h \mathrm{Mpc}^{-1}\right) \leq 1$, with a smoothing scale of $M_{\text {smooth }}=10^{14} M_{\odot}$. Fig. 1 shows the resulting unmarginalized (left panel) and marginalized (right panel) constraints on the parameters $f_{\mathrm{NL}}^{i}$. For both sets of constraints, we first marginalized over the other cosmological parameters. ${ }^{3}$ The $f_{\mathrm{NL}}^{i}$ have most of their degeneracy among themselves; a plot showing the fully unmarginalized constraints on the $f_{\mathrm{NL}}^{i}$ would not look much different than the left panel of Fig. 1. Note that, while some of the $f_{\mathrm{NL}}^{i}$ have support at $k>k_{\max }(z=1) \approx 0.2 \mathrm{~h} \mathrm{Mpc}^{-1}$, we only use information about those (and other) parameters coming from $k<k_{\max }$. The constraints vary considerably as a function of the $k$ at which these parameters are defined. The best-constrained $f_{\mathrm{NL}}^{i}$ corresponds to the $10^{-0.8}<k<10^{-0.6}$ bin, and it has an estimated unmarginalized error of $\sigma\left(f_{\mathrm{NL}}^{16}\right)=7.3$; for comparison, the worst-constrained $f_{\mathrm{NL}}^{i}$, which corresponds to the largest scale (smallest $k$ ) bin, has an unmarginalized error well over one billion.

As expected, the marginalized constraints for the best-constrained parameters are much weaker than the unmarginalized constraints - even the best-measured $f_{\mathrm{NL}}^{i}$ has an estimated marginalized error of $6 \times 10^{2}$. In general, dependence of the constraints on the value of $k$ is determined by two competing factors: as $k$ increases, there is a larger number of modes, each with a smaller signal (given by the smaller nongaussian bias $\Delta b$ ). The best-constrained $k$ is also affected by the fact that only information out to $k=k_{\max }=$ $0.1 \mathrm{~h} \mathrm{Mpc}^{-1}$ is assumed from the galaxy survey. In particular, we have checked that if we unrealistically assume information to be available at all $k$ (instead of at $k<k_{\max }$ ) without modeling the nonlinearities, the unmarginalized constraints on $f_{\mathrm{NL}}^{i}$ improve monotonically with increasing $k$. Therefore, the raw signal-to-noise ratio in $f_{\mathrm{NL}}^{i}$ increases with $k$. To further demonstrate the effect of the choice of $k_{\max }(z)$, we also plotted the errors obtained with the condition $\sigma\left(\pi /\left(2 k_{\max }\right), z\right)=0.15$, which yields $k_{\max }(z=0) \approx 0.03$.

The smoothing mass scale chosen for this analysis (see Eq. (2.5)) has a small but noticeable effect on the constraints yielded. Figure 2 shows that, in the case of the unmarginalized errors, the $k$ at which non-Gaussianity is best constrained decreases as the smoothing mass scale increases. (The behavior of the marginalized errors is more complicated due to correlations in errors between neighboring $f_{\mathrm{NL}}^{i}$.) Since the mass scale is proportional to the physical scale (to the third power), this means that best-constrained $k$ decreases with increasing smoothing scale $R$, which is exactly what we should expect. We remind the reader that while a survey filtered at some scale $M_{\text {smooth }}$ contains objects roughly more massive than this scale, in practice the near-exponentially falling mass function implies that the number density is dominated with $M \simeq M_{\text {smooth }}$ halos.

\footnotetext{
${ }^{3}$ Using six cosmological parameters along with five $b_{0}(z)$ and $20 f_{\mathrm{NL}}^{i}$ led us into some issues with floatingpoint errors and numerical precision. The $31 \times 31$ Fisher matrix we obtained was rather ill-conditioned and difficult to invert reliably using 64-bit precision; we were eventually forced to move to 128-bit precision in order to accurately marginalize over the cosmological parameters.
} 


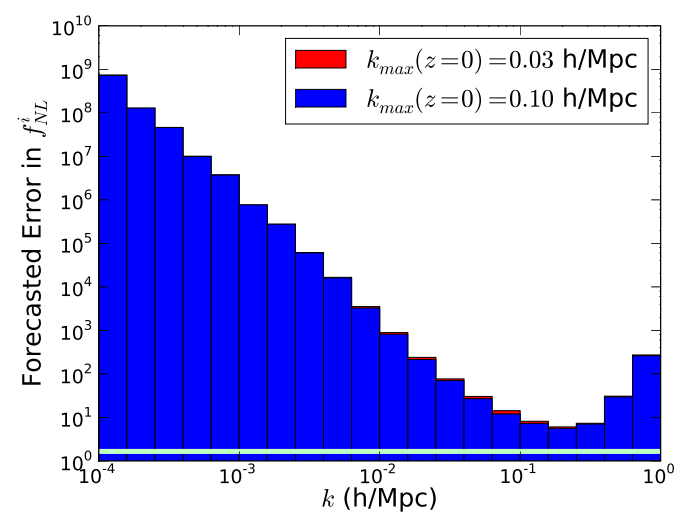

(a) Unmarginalized errors

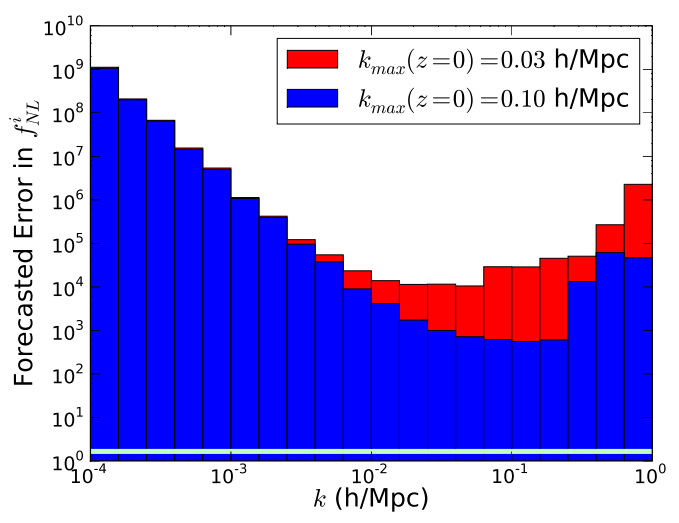

(b) Marginalized errors

Figure 1: Estimated unmarginalized (left panel) and marginalized (right panel) constraints on piecewise-constant parameters $f_{\mathrm{NL}}^{i}$ assuming a future galaxy survey covering one-quarter of the sky out to $z=1$, with average number density of $2 \times 10^{-4}$ gal $/ \mathrm{Mpc}^{3}$. For comparison, the green line is the constraint found for a constant $f_{\mathrm{NL}}$ using the same survey assumptions, and the red histograms are the constraints found with a lower $k_{\max }$ (see text for details). While the individual parameters $f_{\mathrm{NL}}^{i}$ are poorly constrained as expected, their few best linear combinations - the principal components - are well measured; see the next section and text for details.

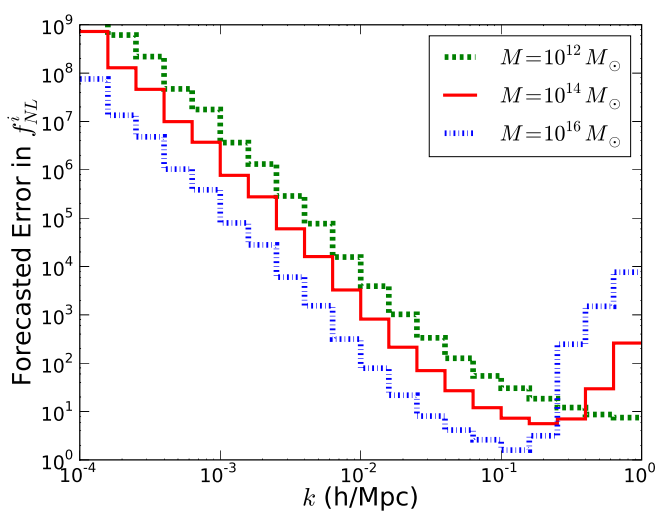

(a) Unmarginalized errors

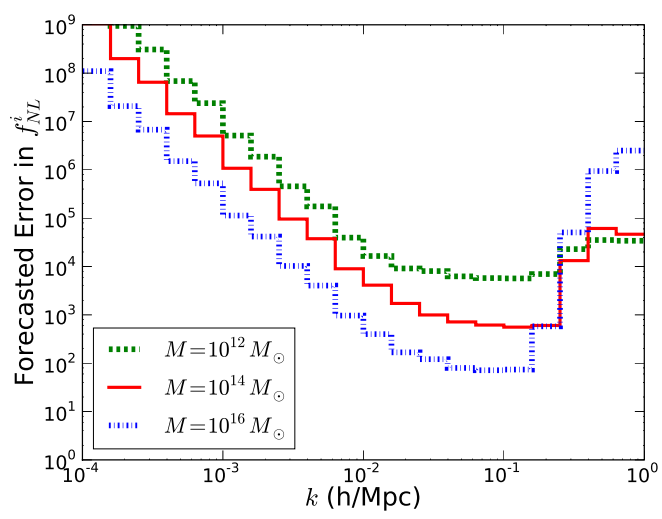

(b) Marginalized errors

Figure 2: Estimated constraints obtained from future surveys with the same parameters as the previous figure at different mass smoothing scales $M_{\text {smooth }}$ (labeled as $M$ in the legend). In other words, these are errors for a survey with halos of $M \gtrsim M_{\text {smooth }}$.

\section{Projection and Principal Components}

\subsection{Constraining other $f_{\mathrm{NL}}(k)$ models}

Once the Fisher matrix $F$ has been obtained for the set of parameters $f_{\mathrm{NL}}^{i}$, it is quite simple to find the best possible constraints on the $f_{\mathrm{NL}}^{i}$ that could be obtained from a future galaxy 
redshift survey. By projecting this Fisher matrix into another basis (see Appendix A), it is also possible to find the constraints on any arbitrary $f_{\mathrm{NL}}(k)$ without calculating a new Fisher matrix from scratch. A trivial example can be found in Appendix A, where we find that the estimated error on a constant $f_{\mathrm{NL}}$, assuming the same future survey as in the previous section, is $\sigma\left(f_{\mathrm{NL}}\right)=2.1$. (Note that this forecasted constraint is on a par with the error expected from Planck, where $\sigma\left(f_{\mathrm{NL}}\right) \sim 5$.)

For another, scale-dependent example, consider the simple form of non-Gaussianity analogous to the conventional parameterization of the power spectrum

$$
f_{\mathrm{NL}}(k)=f_{\mathrm{NL}}^{*}\left(\frac{k}{k_{*}}\right)^{n_{\mathrm{NG}}}
$$

where $k_{*}$ is an arbitrary fixed parameter, leaving $f_{\mathrm{NL}}^{*}$ and $n_{\mathrm{NG}}$ as the parameters of interest in this model. ( $k_{*}$ is generally chosen to minimize degeneracy between $f_{\mathrm{NL}}^{*}$ and $n_{\mathrm{NG}}$ for the observable of interest. We have set $k_{*}=0.165 \mathrm{~h} \mathrm{Mpc}^{-1}$, close to the optimal value in our case; in CMB analysis, the optimal value is lower, around $0.06 h \mathrm{Mpc}^{-1}$.) The partial derivatives of our basis of $f_{\mathrm{NL}}^{i}$ with respect to these parameters are:

$$
\begin{aligned}
\frac{\partial f_{\mathrm{NL}}^{i}}{\partial f_{\mathrm{NL}}^{*}} & =\left(\frac{k}{k_{*}}\right)^{n_{\mathrm{NG}}} ; \\
\frac{\partial f_{\mathrm{NL}}^{i}}{\partial n_{\mathrm{NG}}} & =f_{\mathrm{NL}}^{*}\left(\frac{k}{k_{*}}\right)^{n_{\mathrm{NG}}} \log \left(\frac{k}{k_{*}}\right) .
\end{aligned}
$$

Starting in a basis of $20 f_{\mathrm{NL}}^{i}$ evenly spaced in $\log k$, we project down to a basis of $f_{\mathrm{NL}}^{*}$ and $n_{\mathrm{NG}}$ in order to forecast constraints on the two new parameters from a survey covering onequarter of the sky out to $z=1$. We are using the same limits of integration as in Section 4.1, along with the fiducial values $f_{\mathrm{NL}}^{*}=50$ and $n_{\mathrm{NG}}=0$. The forecasted constraints on these parameters, marginalized over each other, are $\sigma_{f_{\mathrm{NL}}^{*}}=1.7$ and $\sigma_{n_{\mathrm{NG}}}=0.58$. Despite a superficial similarity between this model and the model used by Sefusatti et al. in [23], the two models are quite different, and our results cannot be compared. The model used in [23] is a function of three arguments, $k_{1}, k_{2}$, and $k_{3}$ :

$$
f_{\mathrm{NL}}\left(k_{1}, k_{2}, k_{3}\right)=f_{\mathrm{NL}}^{*}\left(\frac{K}{k_{*}}\right)^{n_{N G}},
$$

where $K=\left(k_{1} k_{2} k_{3}\right)^{1 / 3}$. This leads to a bispectrum of the form found in Eq. (3.8), but with $f_{\mathrm{NL}}\left(k_{1}, k_{2}, k_{3}\right)$ in place of $f_{\mathrm{NL}}$, whereas our bispectrum is of the less-factorizable form Eq. (2.7).

Another example we consider is the form of non-Gaussianity in which the running on $f_{\mathrm{NL}}$ itself has running; that is, the case in which $n_{\mathrm{NG}}$ is a function of $k$. A simple case of this would be $f_{\mathrm{NL}}$ of the form ${ }^{4}$

$$
f_{\mathrm{NL}}(k)=e^{A k^{B}}
$$

Projecting the Fisher matrix down from the original basis $f_{\mathrm{NL}}^{i}$ to the parameters $A$ and $B$, with fiducial values of $A=\log 50$ and $B=0$, we obtain forecasted constraints of $\sigma_{A}=1.0$ and $\sigma_{B}=0.15$. (In this case, the survey characteristics and bounds of integration are the same as in the previous example.)

\footnotetext{
${ }^{4}$ Analogous parameterization for the power spectrum and its motivations are discussed in [64].
} 

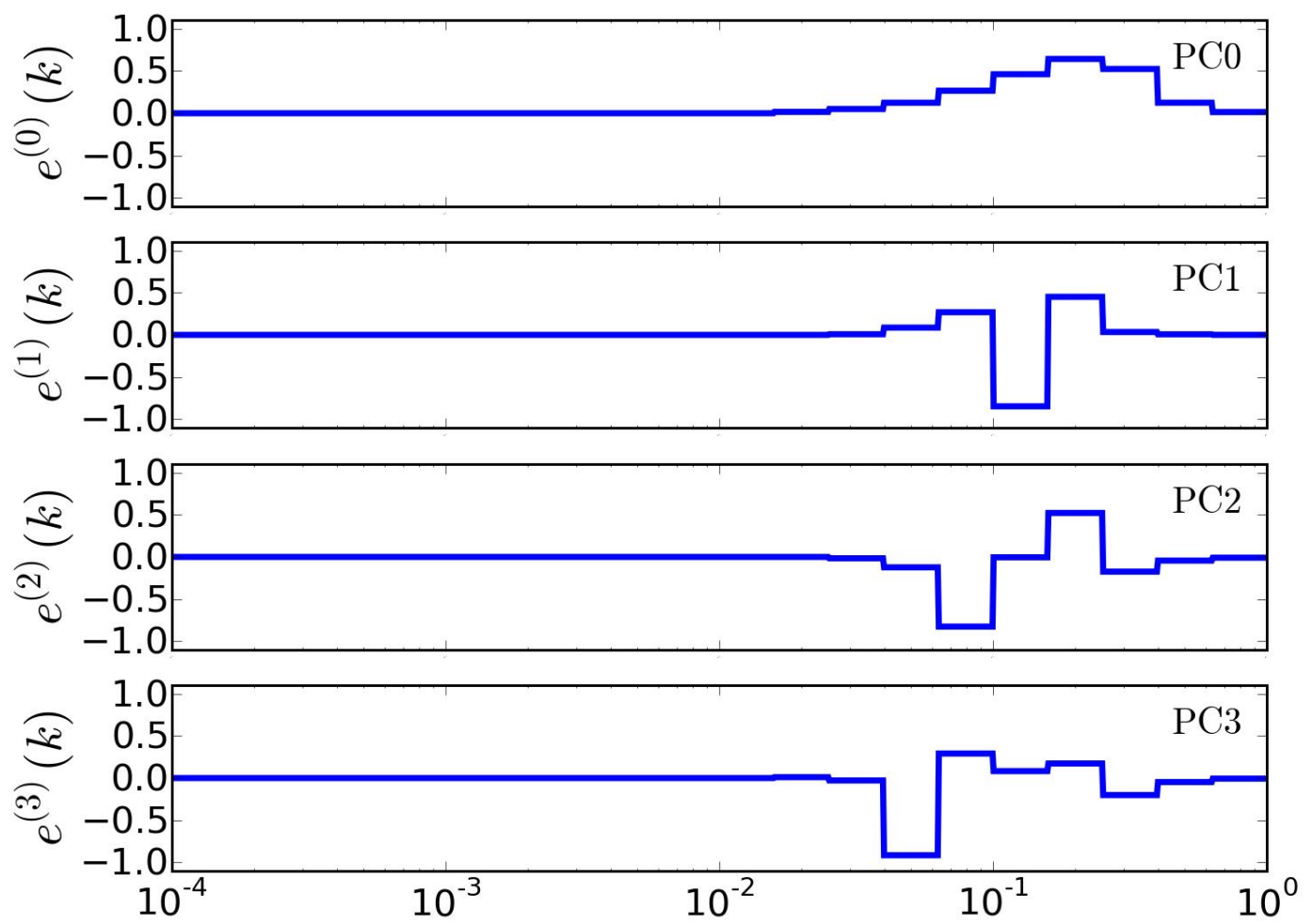

Figure 3: The first four principal components of $f_{\mathrm{NL}}(k)$. The PCs, $e^{(j)}(k)$, are eigenvectors of the Fisher matrix for the $f_{\mathrm{NL}}^{i}$, and are ordered from the best-measured one $(j=0)$ to the worstmeasured one $(j=19)$ for the assumed fiducial survey.

\subsection{Principal components and relation to local and equilateral models}

We now represent a general function $f_{\mathrm{NL}}(k)$ in terms of principal components (PCs). In this approach, the data determine which particular modes of $f_{\mathrm{NL}}(k)$ are best or worst measured. The PCs also constitute a useful form of data compression, so that one can keep only a few of the best-measured modes to make inferences about the function $f_{\mathrm{NL}}(k)$. Finally, the PCs will also enable us to measure the degree of similarity between our scale-dependent ansatz and the local and equilateral forms of non-Gaussianity.

It is rather straightforward to start from the covariance matrix for the piecewise constant parameters $f_{\mathrm{NL}}^{i}$ and obtain the PCs of $f_{\mathrm{NL}}(k)$. The PCs are weights in wavenumber with amplitudes that are uncorrelated by construction, and they are ordered from the best-measured $(i=0)$ to the worst-measured $(i=19)$ for the assumed fiducial survey. The construction of the PCs is described in Appendix B. A few of these PCs of $f_{\mathrm{NL}}(k)$ are shown in Fig. 3. For example, the best-measured $\mathrm{PC}$ has most of its weight around $k=10^{-0.4} h \mathrm{Mpc}^{-1}$, which agrees with sensitivities of piecewise-constant parameters shown in Fig. 1. The sensitivity is not greatest at the largest value of $k\left(1 h \mathrm{Mpc}^{-1}\right)$ because we assumed cosmological information from $k \leq k_{\max }=0.1 \mathrm{~h} \mathrm{Mpc}^{-1}$. We checked that information available at a higher $k_{\max }$ would shift the "sweet spot" of sensitivity to higher wavenumbers. 


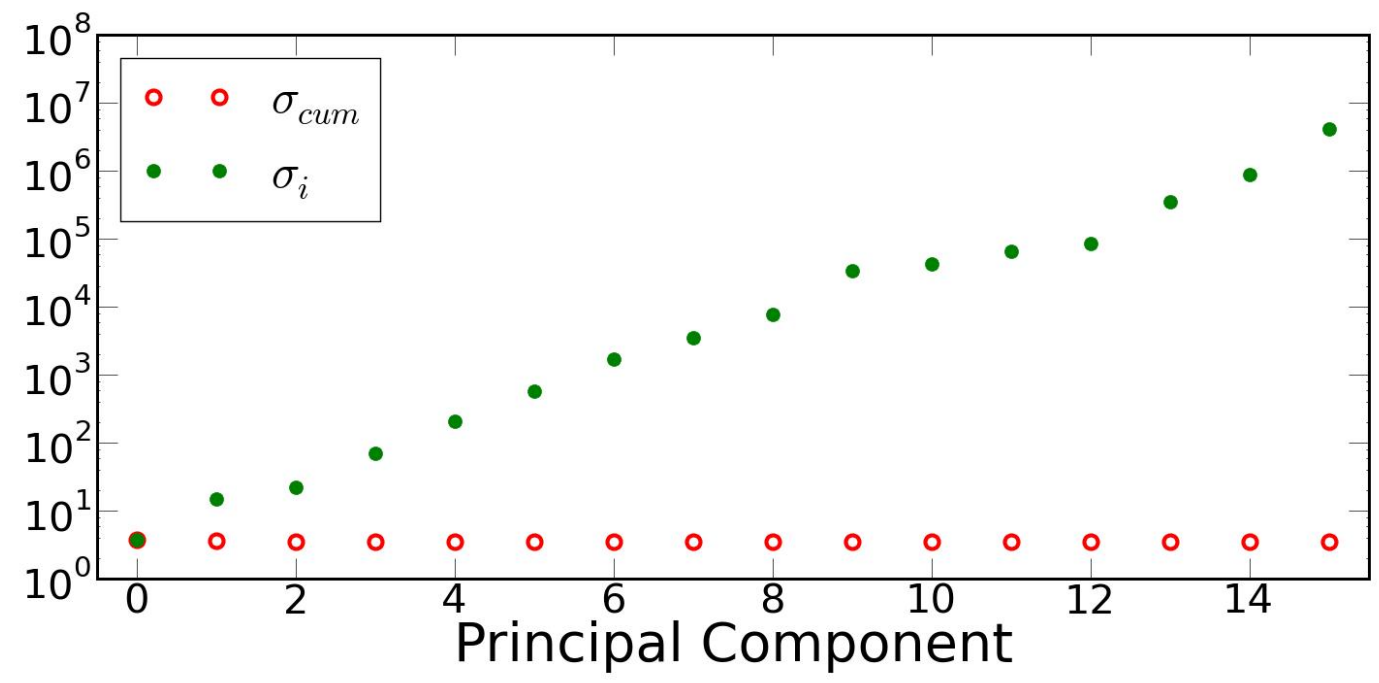

Figure 4: RMS error on each principal component, along with the cumulative error.

The error in the best-measured PC is 4.8; however, the error in the next-best measured PCs are 18.3 and 27.4, and the accuracy rapidly drops off from there. Thus, the first three or four PCs should be enough for any conceivable application. The error in each PC is plotted on a logarithmic scale in figure 4 , along with the cumulative error $\sigma_{\text {cum }}$, which is defined as

$$
\frac{1}{\sigma_{\text {cum }}^{2}}=\sum_{i} \frac{1}{\sigma_{i}^{2}}
$$

Each PC $e^{(j)}(k)$ has its own associated bispectrum (see Eq. (2.7)):

$$
B^{(j)}\left(k_{1}, k_{2}, k_{3}\right)=2\left[e^{(j)}\left(k_{1}\right) P\left(k_{2}\right) P\left(k_{3}\right)+e^{(j)}\left(k_{3}\right) P\left(k_{1}\right) P\left(k_{2}\right)+e^{(j)}\left(k_{2}\right) P\left(k_{3}\right) P\left(k_{1}\right)\right] .
$$

(As always, $k_{1}, k_{2}$, and $k_{3}$ have a triangle relation: $k_{3}=\left|\overrightarrow{k_{2}}-\overrightarrow{k_{1}}\right|$.) We would like to test the similarity of these bispectra to those that have already been discussed in the literature. We can do this by using a distance measure between bispectra, defined by 'cosines' developed in [40]. A cosine near unity implies that the two bispectra have very similar shapes, and a cosine near zero implies the opposite. The cosine is defined as

$$
\cos \left(B_{1}, B_{2}\right)=\frac{B_{1} \cdot B_{2}}{\sqrt{\left(B_{1} \cdot B_{1}\right)\left(B_{2} \cdot B_{2}\right)}},
$$

where the inner product between two bispectra, $B_{1} \cdot B_{2}$, is [23]

$$
B_{1} \cdot B_{2}=\sum_{k_{1}, k_{2}, k_{3}} \frac{B_{1}\left(k_{1}, k_{2}, k_{3}\right) B_{2}\left(k_{1}, k_{2}, k_{3}\right)}{\Delta^{2} B\left(k_{1}, k_{2}, k_{3}\right)} .
$$

The (Gaussian) variance of the bispectrum is

$$
\Delta^{2} B\left(k_{1}, k_{2}, k_{3}\right)=\frac{1}{N_{T}} P\left(k_{1}\right) P\left(k_{2}\right) P\left(k_{3}\right) \sim \frac{1}{N_{T}}\left(k_{1} k_{2} k_{3}\right)^{-3},
$$




\begin{tabular}{|l|c|c|}
\hline & Local cosine & Equilateral cosine \\
\hline$B^{(0)}$ & 0.669 & 0.074 \\
\hline$B^{(1)}$ & 0.040 & 0.000 \\
\hline$B^{(2)}$ & 0.099 & 0.030 \\
\hline$B^{(3)}$ & 0.189 & 0.037 \\
\hline
\end{tabular}

Table 1: Cosines of the first four principal-component derived bispectra with the local bispectrum and the equilateral bispectrum. A cosine near unity implies that the two bispectra have very similar shapes, and a cosine near zero implies the opposite. Note that the zeroth PC, which is by far the best measured (see Fig. 4), has a much larger overlap with the local model than with the equilateral, as expected.

where $N_{T}$ is the number of distinct triangular configurations of $k_{1,2,3}$, and $P(k) \sim k^{-3}$ is the primordial curvature perturbation power spectrum. (The overall constant is irrelevant, since it cancels out in Eq. (5.8).)

We first compare our bispectra Eq. (5.7) to the local model with a constant $f_{\mathrm{NL}}$, whose bispectrum is (see Eqs. (2.1) and (3.8))

$$
B_{\text {local }}\left(k_{1}, k_{2}, k_{3}\right) \propto \frac{1}{k_{1}^{3} k_{2}^{3}}+\frac{1}{k_{1}^{3} k_{3}^{3}}+\frac{1}{k_{2}^{3} k_{3}^{3}}
$$

Most of the power of $B_{\text {local }}$ is in so-called "squeezed" triangles, in which one side is much smaller than the other two (comparable) sides, $k_{1}<<k_{2} \approx k_{3}$.

Another form for the bispectrum much discussed in the literature is the "equilateral" bispectrum

$$
B_{\text {equi }}\left(k_{1}, k_{2}, k_{3}\right)=-\frac{2}{\left(k_{2} k_{1} k_{3}\right)^{2}}-B_{\text {local }}\left(k_{1}, k_{2}, k_{3}\right)+\frac{1}{k_{1} k_{2}^{2} k_{3}^{3}}+\frac{1}{k_{3} k_{1}^{2} k_{2}^{3}}+\text { permutations. }
$$

In contrast with $B_{\text {local }}$, most of the power of $B_{\text {equi }}$ is in triangles where $k_{1} \approx k_{2} \approx k_{3}$; hence the name "equilateral".

Table 1 lists the cosines of the first few principal-component derived bispectra with the local bispectrum and the equilateral bispectrum. The form of Eq. (5.7) suggests that the PC-derived bispectra $B^{(j)}$ will have more in common with the local bispectrum than the equilateral one. However, it is initially conceivable that some $e^{(j)}(k)$ might exist which would yield a bispectrum of the form in Eq. (5.12) when substituted into Eq. (5.7) - but in Appendix C, we prove that no such function exists. Thus, the only guarantees for the cosines of the $B^{(j)}$ are that the cosine of $B^{(0)}$ - the bispectrum corresponding to the bestmeasured PC - will be large with the local model, and that none of the $B^{(j)}$ have a very large cosine with the equilateral model. We expect the former because our model looks like the local model; we expect the latter because of the proof in Appendix C. Table 1 bears out this expectation. The small cosines with the equilateral form of non-Gaussianity are also unsurprising because equilateral non-Gaussianity is expected to have a strongly suppressed signal in the non-Gaussian halo bias [57]. 


\section{Conclusions}

In this paper we have suggested a new phenomenological model of primordial nongaussianity by generalizing the local model (parametrized with a constant parameter $f_{\mathrm{NL}}$ ) to a scale-dependent, non-local class of models. There are multiple ways to do this, and our choice was to write the Newtonian potential as

$$
\Phi(x)=\phi_{G}(x)+f_{\mathrm{NL}}(x) *\left(\phi_{G}(x)^{2}-\left\langle\phi_{G}(x)^{2}\right\rangle\right),
$$

where the convolution in real space corresponds to multiplication in $k$-space, featuring an arbitrary function $f_{\mathrm{NL}}(k)$. Explicit calculations show that such a form of the scale dependent $f_{\mathrm{NL}}$ is borne out in inflationary models [11, 14, 37, 38, 39].

We calculated the bispectrum and bias of dark matter halos in this class of models, following the formalism valid for high peaks $[58,59]$. We then specialized in the piecewise-constant (in wavenumber) parametrization of $f_{\mathrm{NL}}(k)$ which, for the case of narrow enough $k$-bins, recovers any arbitrary function. We used forecasted constraints from an intermediate-future galaxy survey to calculate errors on individual parameters $f_{\mathrm{NL}}^{i}$ (see Fig. 1) and briefly studied dependence on the smoothing scale (Fig. 2).

We further calculated the principal components of $f_{\mathrm{NL}}(k)$, and thus identified the best-measured configurations (in wavenumber) of this function (see Fig. 3). While the sensitivity increases with increasing $k$, restricting the survey information to scales where linear perturbation theory is valid imposes a "sweet spot" in sensitivity of $k \sim 0.1 \mathrm{~h} \mathrm{Mpc}^{-1}$. We then calculated the overlap of the best-measured principal components with two familiar classes of non-Gaussian models: local $\left(f_{\mathrm{NL}}=\right.$ const $)$ and equilateral models, using a cosine measure between the bispectra suggested in [40]. We found the expected result: the best measured component overlaps much more with the local model (which our model generalizes) than with the equilateral one.

One immediate utility of our results is an easy adaptation to specific models of nonGaussianity predicted by classes of inflationary models. If one wants to forecast the accuracy with which parameters of a specific model of $f_{\mathrm{NL}}(k)$-style non-Gaussianity will be measured, neither the halo bias nor the Fisher matrix needs to be calculated from scratch. Instead, our formalism makes it possible to obtain these forecasts by performing a simple linear projection to our piecewise-constant model; this procedure is described in Appendix A and illustrated with a few examples.

In future investigations, it will be interesting to consider specific inflationary models, projecting down to specific forms for $f_{\mathrm{NL}}(k)$. It will also be important to test how well the observable effects of scale-dependent non-Gaussianity, studied here using the theoretical ansatz from Eq. (3.2), agree with numerical simulations; the first such investigations, for select specific forms of $f_{\mathrm{NL}}(k)$, are now being done [41]. Finally, it will be interesting to see how one can optimally select objects in the universe (i.e. their mass) to probe information about scale-dependence of non-Gaussianity. While in Fig. 2 we showed scaling of the bestdetermined scale of $f_{\mathrm{NL}}(k)$ with the smoothing mass scale applied to the density field, a more complete analysis might use the Halo Occupation Distribution (HOD) approach to relate the content of dark matter halos to their mass. 


\section{Acknowledgements}

We thank Chris Byrnes and Sarah Shandera for useful discussions, and the anonymous referee for constructive comments. $\mathrm{AB}$ and $\mathrm{DH}$ are supported by DOE OJI grant under contract DE-FG02-95ER40899, NSF under contract AST-0807564, and NASA under contract NNX09AC89G. KK is supported in part by the Michigan Center for Theoretical Physics. DH and KK would like to thank the Aspen Center for Physics where this project germinated, and DH also acknowledges the generous hospitality of Centro de Ciencias de Benasque "Pedro Pascual".

\section{A. Calculating the error on an arbitrary parametrized $f_{\mathrm{NL}}(k)$}

Projecting the constraints from an old set of parameters $f_{\mathrm{NL}}^{i} \equiv f_{\mathrm{NL}}\left(k_{i}\right)(i=1,2, \ldots, N)$ to new parameters (which we can call $q ; j=1,2, \ldots, M$ for some $M$ ) is in principle straightforward. The Fisher matrix in the new parameters, $F^{\text {new }}$, is given by

$$
F_{i, j}^{\text {new }}=\sum_{k, l=1}^{N} \frac{\partial p^{k}}{\partial q^{i}} \frac{\partial p^{l}}{\partial q^{j}} F_{k l}
$$

so that

$$
F^{\text {new }} \equiv \mathcal{P}^{T} F \mathcal{P}
$$

where $\mathcal{P}_{i j}=\partial p^{i} / \partial q^{j}$ is the derivative matrix of old parameters with respect to new.

Let us look at a couple of examples. Projecting to the case

$$
f_{\mathrm{NL}}(k)=f_{\mathrm{NL}}=\mathrm{const}
$$

is particularly easy, since $\mathcal{P}$ is the column vector with $\mathcal{P}_{i 1}=d f_{\mathrm{NL}}^{i} / d f_{\mathrm{NL}}=1$. Then $F_{i j}^{\text {new }}$ is a $1 \times 1$ matrix that quantifies information on $f_{\mathrm{NL}}$, given by

$$
F_{11}^{\text {new }}=\sum_{k, l} F_{k l}
$$

The error on $f_{\mathrm{NL}}$ is of course given simply by $\sigma\left(f_{\mathrm{NL}}\right)=1 / \sqrt{F_{11}^{\text {new }}}$.

Another example is given by the function

$$
f_{\mathrm{NL}}(k)=\left(\frac{k}{k_{0}}\right)^{n_{\mathrm{NG}}}
$$

with two parameters, $k_{0}$ and $n_{\mathrm{NG}}$. Then one can show that (labeling $k_{0} \equiv q_{1}$ and $n_{\mathrm{NG}} \equiv q_{2}$ ):

$$
\begin{aligned}
& \mathcal{P}_{i 1}=-\frac{n_{\mathrm{NG}}}{k_{0}}\left(\frac{k_{i}}{k_{0}}\right)^{n_{\mathrm{NG}}} ; \\
& \mathcal{P}_{i 2}=\ln \left(\frac{k_{i}}{k_{0}}\right)\left(\frac{k_{i}}{k_{0}}\right)^{n_{\mathrm{NG}}} .
\end{aligned}
$$

Then, using Eq. (A.2), one can simply obtain the $2 \times 2$ Fisher matrix in $k_{0}$ and $n_{\mathrm{NG}}$. 


\section{B. Principal Components of $f_{\mathrm{NL}}(k)$}

We now show how to decompose the measurement of $f_{\mathrm{NL}}(k)$ in principal components, which are essentially the eigenmodes of the covariance matrix for the aforementioned parameters $f_{\mathrm{NL}}\left(k_{i}\right)$. This method has been widely used in cosmology, including applications to parametrizing and describing dark energy $[65,66]$. It allows us to order the best-to-worst measured weights in wavenumber of the function $f_{\mathrm{NL}}(k)$.

Let the function $f_{\mathrm{NL}}(k)$ be described in terms of piecewise constant parameters $f_{\mathrm{NL}}^{i} \equiv$ $f_{\mathrm{NL}}\left(k_{i}\right)$, where

$$
f_{\mathrm{NL}}(k)=\sum_{i=1}^{N} p_{i} \Theta_{i}(k) .
$$

Here, $\Theta(k) \equiv\left[H\left(k-k_{i}^{\text {lower }}\right)-H\left(k-k_{i}^{\text {upper }}\right)\right]$ is the top-hat function of unit height over the $i$ th wavenumber bin, and we assume a total of $N$ bins. $k_{i}^{\text {lower }}$ and $k_{i}^{\text {upper }}$ are the wavenumber bin boundaries, and $H$ is the Heaviside step function. We have effectively expanded the function around the zero value, though this is not crucial: the left-hand side could be $f_{\mathrm{NL}}(k)-f_{\mathrm{NL}}^{\mathrm{fid}}(k)$, for any fiducial $f_{\mathrm{NL}}^{\mathrm{fid}}(k)$, and the formalism still follows.

The Fisher matrix $F$ is the inverse covariance matrix in the original piecewise-constant parameters $p_{i}$, so that $F_{i j}^{-1}=\left\langle p_{i} p_{j}\right\rangle-\left\langle p_{i}\right\rangle\left\langle p_{j}\right\rangle$. We first diagonalize the Fisher matrix $F$ :

$$
F=W^{T} D W,
$$

where $D$ is diagonal and $W$ is some orthogonal matrix. The vector of uncorrelated parameters, $\mathbf{q}$, is related to the vector of original parameters $\mathbf{p}$ via

$$
\mathbf{q}=W \mathbf{p}
$$

and it is easy to check that the $\mathbf{q}$ are uncorrelated; that is, $\left\langle\mathbf{q} \mathbf{q}^{T}\right\rangle=D^{-1}$. The rows of $W$ are therefore the new parameters.

Thus, to calculate the principal components:

1. Obtain the full Fisher matrix for $N$ parameters $p_{i}$, plus the cosmological parameters $\Omega_{b} h^{2}, \Omega_{C D M} h^{2}, H_{0}, w, \log A_{s}$, and $n_{s}$.

2. Marginalize over the cosmological parameters by inverting this larger Fisher matrix, taking the $N \times N$ submatrix, then inverting back to get the Fisher matrix of the $p_{i}$; we call this Fisher matrix $F$

3. Diagonalize $F$ as in Eq. (B.2)

4. The rows of $W$ are the principal components. More precisely, $q_{a}=\sum_{i} W_{a i} p_{i}$, and $q_{a}$ are the PCs.

Let us now change notation slightly (to agree with the commonly used one, e.g. [65]), and define the shape of the $a$-th principal component in $i$-th redshift bin as $\alpha_{i}^{(a)}$, so that 
$\alpha_{i}^{(a)} \equiv W_{a i}$. Then we can represent the $a$-th principal component, $e^{(a)}(k)$, in terms of the original parameters $p_{i}$ as $^{5}$

$$
e^{(a)}(k)=\sum_{i=1}^{N} \alpha_{i}^{(a)} p_{i} \Theta_{i}(k) .
$$

The PCs are obviously uncorrelated, and their eigenvalues $\lambda_{a}$, so that

$$
\left\langle e^{(a)} e^{(b)}\right\rangle \equiv \sum_{i, j=1}^{N} \alpha_{i}^{(a)} \alpha_{j}^{(b)}\left\langle p_{i} p_{j}\right\rangle=\frac{\delta_{a b}}{\lambda_{a}} .
$$

where, recall, $\lambda_{a} \equiv D_{a a}$.

Finally, let us calculate the coefficients $c^{(a)}$ in the expansion in principal components of an arbitrary $f_{\mathrm{NL}}(k)$

$$
f_{\mathrm{NL}}(k)=\sum_{a=1}^{N} c_{a} e^{(a)}(k) .
$$

Let coefficients $f_{\mathrm{NL}}^{i}$ describe $f_{\mathrm{NL}}(k)$ in our original basis, so that $f_{\mathrm{NL}}(k)=$ const $\equiv$ $\sum_{i} f_{\mathrm{NL}}^{i} p_{i} \Theta_{i}(k)$, with $f_{\mathrm{NL}}^{i}$ being left arbitrary for now. Then, taking the expectation value of the product with $e^{(b)}$, we get

$$
\begin{aligned}
\left\langle f_{\mathrm{NL}}(k) e^{(b)}\right\rangle \equiv \frac{c_{b}}{\lambda_{b}} & =\left\langle\left(\sum_{i=1}^{N} f_{\mathrm{NL}}^{i} p_{i}\right) \times\left(\sum_{j=1}^{N} \alpha_{j}^{(a)} p_{j}\right)\right\rangle \\
& =\sum_{i, j=1}^{N} f_{\mathrm{NL}}^{i} \alpha_{j}^{(a)}\left(F^{-1}\right)_{i j},
\end{aligned}
$$

so that

$$
c_{a}=\lambda_{a} \sum_{i, j=1}^{N} f_{\mathrm{NL}}^{i} \alpha_{j}^{(a)}\left(F^{-1}\right)_{i j} .
$$

For example, in the simplest case of constant $f_{\mathrm{NL}}(k)$, where $f_{\mathrm{NL}}^{i}=$ const $\equiv f_{\mathrm{NL}}$, the coefficients of the principal components in the expansion of $f_{\mathrm{NL}}(k)$ are

$$
c_{a}=\lambda_{a} f_{\mathrm{NL}} \sum_{i j} \alpha_{j}^{(a)}\left(F^{-1}\right)_{i j} \quad\left(\text { for } f_{\mathrm{NL}}(k) \equiv f_{\mathrm{NL}}=\text { const }\right) .
$$

\section{Generalized local ansatz does not recover the equilateral case}

Here, we prove that our ansatz cannot perfectly mimic the equilateral bispectrum for any choice of $f_{\mathrm{NL}}(k)$. The generalized local form of the bispectrum that we considered in this paper is

$$
B_{\text {gener }}\left(k_{1}, k_{2}, k_{3}\right)=2\left[f_{\mathrm{NL}}\left(k_{1}\right) P\left(k_{2}\right) P\left(k_{3}\right)+\text { permutations }\right] \propto \frac{f_{\mathrm{NL}}\left(k_{1}\right)}{k_{2}^{3} k_{3}^{3}}+\text { perm } .
$$

\footnotetext{
${ }^{5}$ This is basically the continuous version of the relation $q_{a}=\sum_{i} W_{a i} p_{i}$.
} 
The equilateral bispectrum is

$$
B_{\text {equi }}\left(k_{1}, k_{2}, k_{3}\right) \propto\left[\frac{1}{k_{1} k_{2}^{2} k_{3}^{3}}+\text { perm. }\right]-\frac{2}{\left(k_{2} k_{1} k_{3}\right)^{2}}-\left[\frac{1}{k_{2}^{3} k_{3}^{3}}+\text { perm. }\right] .
$$

The claim is that there is no $f_{\mathrm{NL}}(k)$ such that $B_{\text {gener }}=B_{\text {equi }}$ for all $k_{1}, k_{2}, k_{3}$. To show this, we define a new function $h(k) \equiv f_{\mathrm{NL}}(k)+1$. If there is some $f_{\mathrm{NL}}(k)$ such that $B_{\text {gener }}=B_{\text {equi }}$, then we have:

$$
\frac{h\left(k_{1}\right)}{k_{2}^{3} k_{3}^{3}}+\text { perm. } \propto\left[\frac{1}{k_{1} k_{2}^{2} k_{3}^{3}}+\text { perm. }\right]-\frac{2}{\left(k_{2} k_{1} k_{3}\right)^{2}} .
$$

We can go from a proportionality to an equality by defining a new function $g(k)$ that is simply $h(k)$ with the appropriate constant out in front. Next, multiply both sides by $k_{1}^{3} k_{2}^{3} k_{3}^{3}$ to get

$$
k_{1}^{3} g\left(k_{1}\right)+k_{2}^{3} g\left(k_{2}\right)+k_{3}^{3} g\left(k_{3}\right)=\left[k_{1} k_{2}^{2}+k_{2} k_{3}^{2}+\text { perm. }\right]-2 k_{1} k_{2} k_{3} .
$$

Each term on the left-hand side is dependent on only one of $k_{1}, k_{2}$, or $k_{3}$. However, every term on the right-hand side depends on at least two different $k$; thus, there is no $g(k)$ that can satisfy this relation.

Alternatively, consider the case where $k_{1}=k_{2}=k_{3}=k$. Then (C.3) becomes

$$
3 k^{3} g(k)=4 k^{3}
$$

which means that

$$
g(k)=4 / 3 .
$$

This answer is wholly independent of $k$, so this value of $g(k)$ must be true for all $k$. But this solution for $g(k)$ is clearly incorrect in the general case where $k_{1} \neq k_{2} \neq k_{3}$; therefore, no such $g(k)$ can exist.

While this proves that there is no $f_{\mathrm{NL}}(k)$ that yields an exact equality between our ansatz and the equilateral bispectrum, the question of an approximate equality remains. Such solutions for $f_{\mathrm{NL}}(k)$ certainly exist for narrow ranges of $k$. For example, $f_{\mathrm{NL}}(k)=$ $\delta\left(k-k^{*}\right)$, where $\delta(k)$ is the Dirac delta function, yields a bispectrum that is larger for exactly one equilateral triangle - the triangle where $k_{1,2,3}=k_{*}$ - than it is for any squeezed triangle. However, no $f_{\mathrm{NL}}(k)$ exists that yields a bispectrum which favors equilateral triangles over squeezed triangles for all $k$. It is straightforward but tedious to prove this fact, and the details of the proof are beyond the scope of this paper.

\section{References}

[1] J. Maldacena, Non-gaussian features of primordial fluctuations in single field inflationary models, Journal of High Energy Physics 5 (2003) 13, [arXiv:astro-ph/0210603].

[2] V. Acquaviva, N. Bartolo, S. Matarrese, and A. Riotto, Second-order cosmological perturbations from inflation, Nucl. Phys. B667 (2003) 119-148, [astro-ph/0209156]. 
[3] P. Creminelli, On non-gaussianities in single-field inflation, JCAP 10 (2003) 003, [astro-ph/0306122].

[4] D. H. Lyth and Y. Rodriguez, The inflationary prediction for primordial non- gaussianity, Phys. Rev. Lett. 95 (2005) 121302, [astro-ph/0504045].

[5] D. Seery and J. E. Lidsey, Primordial non-gaussianities in single field inflation, JCAP 06 (2005) 003, [astro-ph/0503692].

[6] X. Chen, Primordial Non-Gaussianities from Inflation Models, Adv. Astron. 2010 (2010) 638979, [arXiv: 1002.1416].

[7] E. Komatsu, Hunting for Primordial Non-Gaussianity in the Cosmic Microwave Background, Class. Quant. Grav. 27 (2010) 124010, [arXiv:1003.6097].

[8] E. Komatsu and D. N. Spergel, Acoustic signatures in the primary microwave background bispectrum, Phys. Rev. D 63 (Mar., 2001) 063002, [arXiv:astro-ph/0005036].

[9] L. Verde, L.-M. Wang, A. Heavens, and M. Kamionkowski, Large-scale structure, the cosmic microwave background, and primordial non-gaussianity, Mon. Not. Roy. Astron. Soc. 313 (2000) L141-L147, [astro-ph/9906301].

[10] R. Scoccimarro, E. Sefusatti, and M. Zaldarriaga, Probing primordial non-gaussianity with large-scale structure, Phys. Rev. D 69 (2004) 103513, [astro-ph/0312286].

[11] D. Salopek and J. Bond, Nonlinear evolution of long wavelength metric fluctuations in inflationary models, Phys.Rev. D 42 (1990) 3936-3962.

[12] T. Falk, R. Rangarajan, and M. Srednicki, The angular dependence of the three point correlation function of the cosmic microwave background radiation as predicted by inflationary cosmologies, Astrophys. J. 403 (1993) L1, [astro-ph/9208001].

[13] X.-c. Luo and D. N. Schramm, Testing for the gaussian nature of cosmological density perturbations through the three-point temperature correlation function, Phys. Rev. Lett. 71 (1993) 1124-1127, [astro-ph/9305009].

[14] A. Gangui, F. Lucchin, S. Matarrese, and S. Mollerach, The three point correlation function of the cosmic microwave background in inflationary models, Astrophys. J. 430 (1994) 447-457, [astro-ph/9312033].

[15] L.-M. Wang and M. Kamionkowski, The cosmic microwave background bispectrum and inflation, Phys. Rev. D 61 (2000) 063504, [astro-ph/9907431].

[16] N. Bartolo, E. Komatsu, S. Matarrese, and A. Riotto, Non-gaussianity from inflation: Theory and observations, Phys. Rept. 402 (2004) 103-266, [astro-ph/0406398].

[17] D. Seery and J. E. Lidsey, Primordial non-Gaussianities from multiple-field inflation, JCAP 0509 (2005) 011, [astro-ph/0506056].

[18] X. Chen, Running Non-Gaussianities in DBI Inflation, Phys. Rev. D 72 (2005) 123518, [astro-ph/0507053].

[19] M. Liguori, F. K. Hansen, E. Komatsu, S. Matarrese, and A. Riotto, Testing primordial non-Gaussianity in CMB anisotropies, Phys. Rev. D 73 (Feb., 2006) 043505, [arXiv:astro-ph/0509098].

[20] X. Chen, R. Easther, and E. A. Lim, Large non-Gaussianities in single field inflation, JCAP 06 (2007) 023, [astro-ph/0611645]. 
[21] M. LoVerde, A. Miller, S. Shandera, and L. Verde, Effects of Scale-Dependent Non-Gaussianity on Cosmological Structures, JCAP 04 (2008) 014, [arXiv:0711.4126].

[22] X. Chen, R. Easther, and E. A. Lim, Generation and Characterization of Large Non-Gaussianities in Single Field Inflation, JCAP 04 (2008) 010, [arXiv:0801.3295].

[23] E. Sefusatti, M. Liguori, A. P. S. Yadav, M. G. Jackson, and E. Pajer, Constraining running non-gaussianity, JCAP 12 (Dec., 2009) 22, [arXiv:0906.0232].

[24] J. Kumar, L. Leblond, and A. Rajaraman, Scale dependent local non-gaussianity from loops, JCAP 04 (Apr., 2010) 24, [arXiv: 0909. 2040].

[25] C. T. Byrnes, S. Nurmi, G. Tasinato, and D. Wands, Scale dependence of local $f_{N L}, J C A P$ 02 (Feb., 2010) 34, [arXiv:0911.2780].

[26] C. T. Byrnes and K.-Y. Choi, Review of local non-Gaussianity from multi-field inflation, Adv. Astron. 2010 (2010) 724525, [arXiv:1002.3110].

[27] D. Wands, Local non-Gaussianity from inflation, Class.Quant.Grav. 27 (2010) 124002, [arXiv: 1004.0818].

[28] A. Riotto and M. S. Sloth, Strongly Scale-dependent Non-Gaussianity, arXiv:1009.3020.

[29] Q.-G. Huang, Scale dependence of $f_{N L}$ in $N$-flation, arXiv:1009.3326.

[30] S. Mollerach, Isocurvature Baryon Perturbations and Inflation, Phys.Rev. D 42 (1990) 313-325.

[31] A. D. Linde and V. F. Mukhanov, Nongaussian isocurvature perturbations from inflation, Phys.Rev. D 56 (1997) 535-539, [astro-ph/9610219].

[32] K. Enqvist and M. S. Sloth, Adiabatic CMB perturbations in pre - big bang string cosmology, Nucl.Phys. B 626 (2002) 395-409, [hep-ph/0109214].

[33] D. H. Lyth and D. Wands, Generating the curvature perturbation without an inflaton, Phys. Lett. B 524 (2002) 5-14, [hep-ph/0110002].

[34] T. Moroi and T. Takahashi, Effects of cosmological moduli fields on cosmic microwave background, Phys.Lett. B 522 (2001) 215-221, [hep-ph/0110096].

[35] L. Kofman, Probing string theory with modulated cosmological fluctuations, astro-ph/0303614.

[36] M. Zaldarriaga, Non-Gaussianities in models with a varying inflaton decay rate, Phys.Rev. D69 (2004) 043508, [astro-ph/0306006].

[37] C. T. Byrnes, M. Gerstenlauer, S. Nurmi, G. Tasinato, and D. Wands, Scale-dependent non-Gaussianity probes inflationary physics, JCAP 10 (2010) 004, [arXiv:1007.4277].

[38] C. T. Byrnes, K. Enqvist, and T. Takahashi, Scale-dependence of Non-Gaussianity in the Curvaton Model, JCAP 09 (2010) 026, [arXiv: 1007.5148].

[39] Q.-G. Huang, Negative spectral index of $f_{N L}$ in the axion-type curvaton model, JCAP 11 (2010) 026, [arXiv: 1008.2641].

[40] D. Babich, P. Creminelli, and M. Zaldarriaga, The shape of non-gaussianities, JCAP 08 (2004) 009, [astro-ph/0405356].

[41] S. Shandera, N. Dalal, and D. Huterer, A generalized local ansatz and its effect on halo bias, arXiv: 1010.3722 . 
[42] N. Dalal, O. Dore, D. Huterer, and A. Shirokov, The imprints of primordial non-gaussianities on large- scale structure: scale dependent bias and abundance of virialized objects, Phys. Rev. D77 (2008) 123514, [arXiv:0710.4560].

[43] N. Afshordi and A. J. Tolley, Primordial non-gaussianity, statistics of collapsed objects, and the Integrated Sachs-Wolfe effect, Phys. Rev. D78 (2008) 123507, [arXiv:0806.1046].

[44] S. Matarrese and L. Verde, The effect of primordial non-Gaussianity on halo bias, Astrophys. J. 677 (2008) L77, [arXiv:0801.4826].

[45] A. Slosar, C. Hirata, U. Seljak, S. Ho, and N. Padmanabhan, Constraints on local primordial non-Gaussianity from large scale structure, JCAP 08 (2008) 031, [arXiv:0805.3580].

[46] F. Schmidt and M. Kamionkowski, Halo Clustering with Non-Local Non-Gaussianity, Phys. Rev. D 82 (2010) 103002, [arXiv:1008.0638].

[47] P. McDonald, Primordial non-Gaussianity: large-scale structure signature in the perturbative bias model, Phys. Rev. D78 (2008) 123519, [arXiv:0806.1061].

[48] A. Taruya, K. Koyama, and T. Matsubara, Signature of primordial non-Gaussianity on the matter power spectrum, Phys. Rev. D 78 (Dec., 2008) 123534, [arXiv:0808.4085].

[49] T. Giannantonio and C. Porciani, Structure formation from non-Gaussian initial conditions: multivariate biasing, statistics, and comparison with $N$ - body simulations, Phys. Rev. D81 (2010) 063530, [arXiv:0911.0017].

[50] M. Grossi, K. Dolag, E. Branchini, S. Matarrese, and L. Moscardini, Evolution of Massive Haloes in non-Gaussian Scenarios, Mon. Not. Roy. Astron. Soc. 382 (July, 2007) 1261, [arXiv:0707.2516].

[51] V. Desjacques, U. Seljak, and I. T. Iliev, Scale-dependent bias induced by local non-Gaussianity: a comparison to N-body simulations, MNRAS 396 (June, 2009) 85-96, [arXiv:0811.2748].

[52] A. Pillepich, C. Porciani, and O. Hahn, Halo mass function and scale-dependent bias from N-body simulations with non-Gaussian initial conditions, MNRAS 402 (Feb., 2010) 191-206, [arXiv: 0811.4176].

[53] C. Carbone, L. Verde, and S. Matarrese, Non-Gaussian halo bias and future galaxy surveys, Astrophys. J. 684 (2008) L1-L4, [arXiv:0806.1950].

[54] B. Sartoris et al., The potential of X-ray cluster surveys to constrain primordial non-Gaussianity, arXiv:1003.0841.

[55] C. Cunha, D. Huterer, and O. Doré, Primordial non-gaussianity from the covariance of galaxy cluster counts, Phys. Rev. D 82 (Jul, 2010) 023004, [arXiv:1003.2416].

[56] V. Desjacques and U. Seljak, Signature of primordial non-Gaussianity of $\phi^{3}$-type in the mass function and bias of dark matter haloes, Phys. Rev. D81 (2010) 023006, [arXiv:0907.2257].

[57] L. Verde and S. Matarrese, Detectability of the effect of Inflationary non-Gaussianity on halo bias, Astrophys. J. 706 (2009) L91-L95, [arXiv:0909.3224].

[58] B. Grinstein and M. B. Wise, Nongaussian Fluctuations and the Correlations of Galaxies or Rich Clusters of Galaxies, Astrophys. J. 310 (1986) 19-22.

[59] S. Matarrese, F. Lucchin, and S. A. Bonometto, A path-integral approach to large-scale matter distribution originated by non-Gaussian fluctuations, Astrophys. J. 310 (Nov., 1986) L21-L26. 
[60] H. A. Feldman, N. Kaiser, and J. A. Peacock, Power spectrum analysis of three-dimensional redshift surveys, Astrophys. J. 426 (1994) 23-37, [astro-ph/9304022].

[61] M. Tegmark, Measuring cosmological parameters with galaxy surveys, Phys. Rev. Lett. 79 (1997), no. 20 3806-3809, [astro-ph/9706198].

[62] H. Seo and D. J. Eisenstein, Probing Dark Energy with Baryonic Acoustic Oscillations from Future Large Galaxy Redshift Surveys, Astrophys. J. 598 (Dec., 2003) 720-740, [arXiv : astro-ph/0307460].

[63] E. Komatsu et al., Seven-Year Wilkinson Microwave Anisotropy Probe (WMAP) Observations: Cosmological Interpretation, arXiv:1001.4538.

[64] K. Abazajian, K. Kadota, and E. D. Stewart, Parameterizing the power spectrum: Beyond the truncated Taylor expansion, JCAP 0508 (2005) 008, [astro-ph/0507224].

[65] D. Huterer and G. Starkman, Parameterization of dark-energy properties: A principalcomponent approach, Phys. Rev. Lett. 90 (2003) 031301, [astro-ph/0207517].

[66] A. J. Albrecht et al., Findings of the Joint Dark Energy Mission Figure of Merit Science Working Group, arXiv:0901.0721. 